\title{
Wrapping of a spherical colloid by a fluid membrane
}

\author{
Markus Deserno ${ }^{1}$ and Thomas Bickel ${ }^{1,2}$ \\ ${ }^{1}$ Department of Chemistry and Biochemistry, UCLA, Box 951569, Los Angeles, CA 90095, USA and \\ ${ }^{2}$ Department of Physics and Astronomy, UCLA, Box 951547, Los Angeles, CA 90095, USA
}

(Dated: December 16, 2002)

\begin{abstract}
We theoretically study the elastic deformation of a fluid membrane induced by an adhering spherical colloidal particle within the framework of a Helfrich energy. Based on a full optimization of the membrane shape we find a continuous binding and a discontinuous envelopment transition, the latter displaying a potentially substantial energy barrier. A small gradient approximation permits membrane shape and complex energy to be calculated analytically. While this only leads to a good representation of the complex geometry for very small degrees of wrapping, it still gives the correct phase boundaries in the regime of low tension.
\end{abstract}

\section{INTRODUCTION AND MOTIVATION}

Fluid lipid bilayers are one of the key structural elements of all living cells. On the one hand they partition and thereby organize the complex hierarchy of intracellular biochemical environments. On the other hand they provide controlled transport between neighboring compartments as well as the extracellular space. These transport mechanisms span quite a large range of particle sizes, all the way from sub-nanometer ions (which cross the membrane via protein channels) up to micron sized objects (like bacteria), which are engulfed in largescale membrane deformations occurring during phagocytosis [1]. Generally, such events require metabolic energy and are meticulously controlled by the cell. However, there are also cases where they happen passively as a consequence of generic physical interactions, for instance a sufficiently strong adhesion between the particle about to be transported and the membrane. An important and well studied example is provided by the route along which many animal viruses leave their host cells, namely, via the wrapping and subsequent pinch-off of a pre-assembled viral nucleoprotein capsid at the plasma membrane 22. Other examples, in which membrane deformations following binding to a small object are crucial, include certain gene transfection systems, in which DNA is complexed by positively charged polymers and the resulting condensed globule becomes internalized by the cell in an adhesion-driven invagination process [3]; or a host of modern biophysical experimental techniques, in which for instance microbeads [4 or AFM tips [5] are in contact with a membrane and become partially or fully wrapped.

These biological examples are complemented by more physically oriented experiments on the adsorption of micrometer sized beads onto model lipid bilayers. Studies have for instance focused on the wrapping of a latex bead by a vesicle [6], or on the interaction between several beads via membrane mediated forces [7]. For these non-flat substrates, it is unfortunately difficult to extract detailed information about the membrane shape close to contact, especially for small colloids for which the bending contribution in the wrapping balance becomes more important [8]. It is therefore desirable to have a better theoretical understanding of how physical parameters like bending stiffness, lateral tension, or adhesion strength control the shape of the complex and under which circumstances complete wrapping ensues. This question has been partially addressed in a recent work that considers the adhesion of a cylindrical rod to a membrane and estimates the force required for unbinding [9]. However, the description is limited to the regime of small wrapping, since it relies on a small gradient expansion of the elastic energy. In the present paper we determine the complete structural wrapping behavior of a spherical colloid by numerically solving the full nonlinear differential equations which describe the shape of an elastic fluid membrane under a prescribed lateral tension adhering to a spherical particle with some given strength. These exact results are then compared to analytical calculations employing a small gradient approximation. We find that in the latter case the shape of the complex is only predicted correctly for very small degrees of wrapping. However, the phase boundary toward envelopment is still accurately represented over a somewhat larger range of penetrations, essentially in the regime of low tension.

\section{GENERAL ENERGY CONSIDERATIONS}

We consider a spherical colloid of radius $a$ adsorbed on a deformable surface, as depicted in Fig. 1. Following Helfrich [10], the elastic energy associated with some membrane deformation is, per unit area,

$$
e_{\mathrm{H}}=\sigma+\frac{\kappa}{2}\left(c_{1}+c_{2}\right)^{2},
$$

with $c_{1}$ and $c_{2}$ the local principal curvatures, $\kappa$ the bending modulus, and $\sigma$ the lateral tension. From the two elastic constants we can construct a length, $\lambda$, according to

$$
\lambda=\sqrt{\frac{\kappa}{\sigma}} .
$$

For instance, with a tension of $\sigma \simeq 0.02 \mathrm{dyn} / \mathrm{cm}$ (a value commonly found for cell membranes [11]) and a typical 


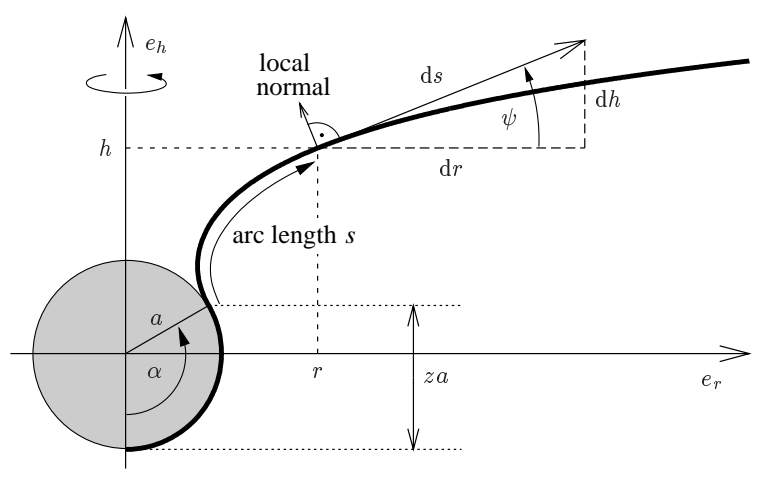

FIG. 1: Geometry of the wrapping complex and membrane parameterization. A membrane adheres partially and cylindrically symmetrically to a spherical colloid of radius $a$ with a degree of wrapping given by $z=1-\cos \alpha$. Due to the possibility of "overhangs" it is advantageous to parameterize the membrane by specifying the angle $\psi$ as a function of arc length $s$. The more direct choice of measuring the height $h$ as a function of radial distance $r$ remains however useful for the small gradient approximation.

bending modulus of $\kappa \simeq 20 k_{\mathrm{B}} T$ (where $k_{\mathrm{B}} T$ is the thermal energy) we obtain $\lambda \simeq 64 \mathrm{~nm}$ (which, incidentally, is about the same size as many viral nucleocapsids [12). Membrane deformations on a length scale smaller than $\lambda$ are mainly controlled by bending energy, while tension is predominant on larger scales. In this study, we will restrict ourselves to the bending-dominated low tension regime, $a \lesssim \lambda$. We will see that even then a comparatively low tension can play an important role.

A natural parameter specifying the coverage of the colloid by the membrane is the degree of wrapping $z=$ $1-\cos \alpha$. It ranges from $z=0$, when the colloid just touches the surface, up to $z=2$, in the fully enveloped state. The equilibrium value of $z$ results from the adhesion of the colloid, driven by a contact energy per unit area, $w$, being balanced by the requirement to bend the membrane as well as the work of pulling excess membrane toward the wrapping site against the prescribed lateral tension $\sigma$.

Energies pertaining to the adhering part can be calculated easily: The adhesion energy equals $-2 \pi a^{2} z w$, while bending and tension contributions are found to be $4 \pi z \kappa$ and $\pi a^{2} z^{2} \sigma$, respectively. The bending and tension energies of the free part of the membrane are more difficult to evaluate, since they require the determination of the equilibrium membrane profile, a task that will be pursued below. Before solving the full variational problem, it is worth noting that the situation is greatly simplified for a membrane with no lateral tension. In this special case the equilibrium profile is quickly seen to be a catenoid, a minimal surface with zero mean curvature. Hence, the only energy contributions stem from the wrapped part of the membrane. We then find that, for $\sigma=0$, colloids do not adhere at low adhesion energy $w<w_{\mathrm{c}}=2 \kappa / a^{2}$, whereas full wrapping occurs above $w_{\mathrm{c}}$, with no energy barrier to be overcome.

\section{NONLINEAR SHAPE EQUATIONS}

As a first approach, it would be tempting to approximate the energy of the free section of the membrane by a phenomenological line energy. However, neither the relation between the line tension constant and the membrane properties $\kappa$ and $\sigma$ would be known, nor is the implied dependency on the degree of wrapping correct. In order to draw indubitable conclusions, it is thus advisable to determine the exact membrane profile.

The energy $E_{\text {free }}$ of the free membrane is the surface integral over the local bending and tension contributions and is thus a functional of the shape. By solving the corresponding Euler-Lagrange equations one obtains the "ground state" profile and thereby its energy. Much work along these lines has for instance led to a detailed understanding of vesicle conformations [13]. In the present context, and using the parameterization indicated in Fig. 1, the shape equations can be written as

$$
\begin{aligned}
\dot{\psi} & =\frac{p_{\psi}}{2 r}-\frac{\sin \psi}{r} \\
\dot{r} & =\cos \psi \\
\dot{h} & =\sin \psi \\
\dot{p}_{\psi} & =\left(\frac{p_{\psi}}{r}-p_{h}\right) \cos \psi+\left(\frac{2 r}{\lambda^{2}}+p_{r}\right) \sin \psi, \\
\dot{p}_{r} & =\frac{p_{\psi}}{r}\left(\frac{p_{\psi}}{4 r}-\frac{\sin \psi}{r}\right)+\frac{2}{\lambda^{2}}(1-\cos \psi) \\
\dot{p}_{h} & =0
\end{aligned}
$$

The dot indicates the derivative with respect to the arc length $s$ along the membrane profile, and the $p$ 's are the "momenta" canonically conjugate to the coordinates $\psi$, $r$, and $h$. These equations are integrated numerically for an asymptotically flat membrane that touches the colloid tangentially at a specific point of detachment. The determination of the appropriate boundary conditions far from the colloid raises a few subtle technical questions, and we refer the reader to Refs. [14] and [15] for further details. From the numerical solution we finally obtain $E_{\text {free }}$ - and thus the total energy - as a function of the degree of wrapping $z$.

The structural phase diagram depicted in Fig. 2 follows directly. We find that the transition from the free to the partially wrapped state is continuous and occurs at the same value $w_{\mathrm{c}}=2 \kappa / a^{2}$ for any value of the tension. In contrast to that, an energy barrier separates the partially wrapped from the the fully enveloped state, rendering this transition discontinuous. The transition lines merge at a triple point $\left(w_{\mathrm{c}}, 0\right)$, as expected. Our approach also allows us to determine the height of the energy barrier (see Fig. 3), which surprisingly originates predominantly from tension. For $\sigma=0.02 \mathrm{dyn} / \mathrm{cm}$ (typical cellular tension [11]), $a=30 \mathrm{~nm}$ (capsid radius of Semliki Forest Virus, an often studied example) and $\kappa=20 k_{\mathrm{B}} T$, the 


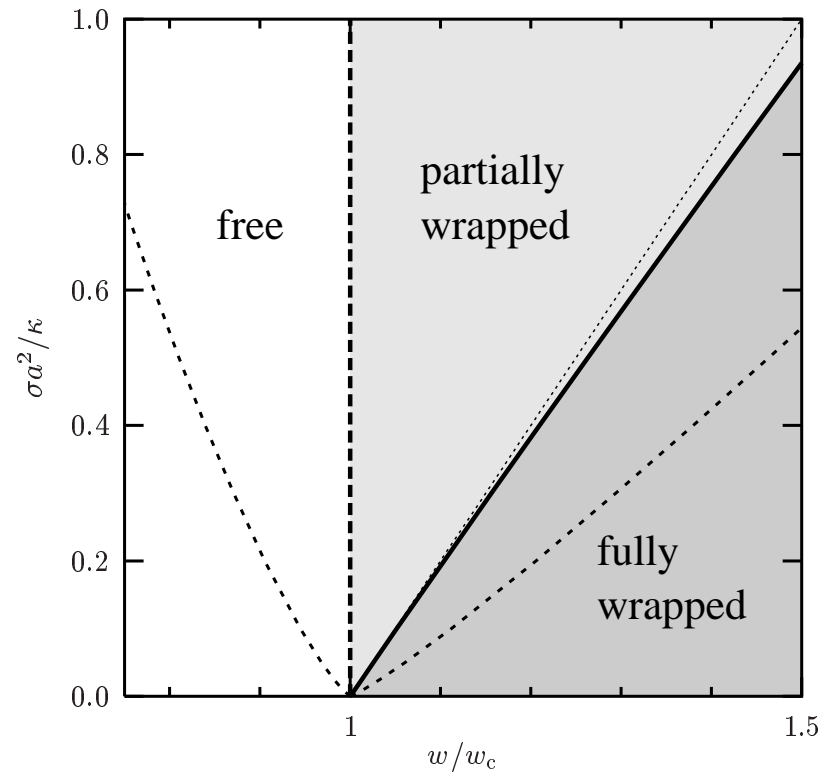

FIG. 2: Structural wrapping phase diagram in the plane of adhesion constant $w$ and lateral tension $\sigma$. The bold solid line indicates the discontinuous transition between partially and fully wrapped, and the short dashed lines are the spinodals belonging to it. The fine dotted line $\sigma=w-w_{\mathrm{c}}$ indicates where the fully enveloped state has zero energy.

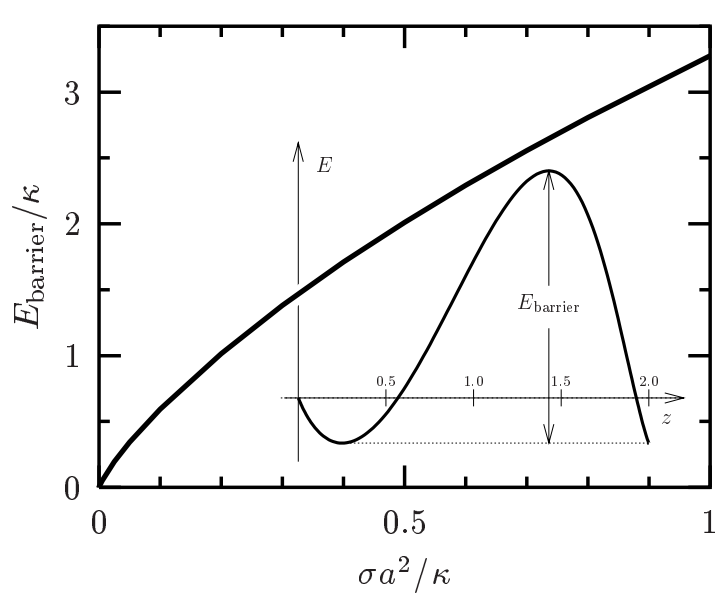

FIG. 3: Height of the energy barrier $E_{\text {barrier }}$ as a function of reduced tension. The inset illustrates the shape of the function $E(z)$ at the value of $w$ where the transition from partial to full wrapping occurs and illustrates the definition of the concomitant energy barrier.

barrier has the substantial value of $E_{\text {barrier }} \approx 22 k_{\mathrm{B}} T$, showing that it cannot be overcome by thermal fluctuations. This point is also illustrated in Fig. 2 by showing the two spinodal lines at which the barrier vanishes. Incidentally, starting with a fully enveloped state and reducing the adhesion $w$, the unwrapping-spinodal is crossed only once one is already in the unbound regime, i.e., the hysteresis would be so pronounced that one "skips" the partially wrapped region upon unbinding.
The energy of the free section of the membrane vanishes in the limit of full wrapping, $z \rightarrow 2$, which is reminiscent of the case of an ideal neck connecting two vesicles [16]. The reason is essentially that as the neck contracts, the necessary membrane back-bending must occur on a length scale much smaller than $\lambda$ and is thus entirely dominated by the bending energy. But then the membrane will just assume the shape of a catenoid which, even though highly deformed, has zero mean curvature and thus does not cost any bending energy.

\section{SMALL GRADIENT EXPANSION, RIGOROUS RESULTS}

The exact shape equations (3) are nonlinear and can only be solved numerically. In order to get analytical information about the triple point at $\left(w_{\mathrm{c}}, 0\right)$, we use a single-valued (Monge-) parameterization of the surface profile $h(\boldsymbol{r})$, where $\boldsymbol{r}=(x, y)$ spans the flat reference plane at $h=0$. This representation does not allow for overhangs and therefore can only describe the first stages of the wrapping process. Assuming moreover that membrane deflections out of the horizontal remain small, i. e., $|\nabla h| \ll 1$, the energy of the free part of the membrane is

$$
E_{\text {free }}=\int \mathrm{d}^{2} \boldsymbol{r}\left\{\frac{\kappa}{2}\left(\nabla^{2} h\right)^{2}+\frac{\sigma}{2}(\nabla h)^{2}\right\} .
$$

This quadratic expansion is valid only for small deformations but it has the advantage to render analytical calculations tractable. The equilibrium profile arises from the stationarity condition $\delta E_{\text {free }} / \delta h=0$ and satisfies the linear Euler-Lagrange equation

$$
\nabla^{2}\left(\nabla^{2}-\lambda^{-2}\right) h=0 .
$$

The general solution of eq. (5) is $h(r)=h_{1}+h_{2} \ln (r / \lambda)+$ $h_{3} K_{0}(r / \lambda)+h_{4} I_{0}(r / \lambda)$, with $K_{0}$ and $I_{0}$ the modified Bessel functions [17]. Far from the colloid, the energy density has to remain finite and integrable, so that $h_{2}=h_{4}=0$. The integration constants $h_{1}$ and $h_{3}$ easily follow from the boundary conditions requiring the profile and its slope to be continuous at the point of detachment. The minimum energy of the functional in eq. (4) is then found to be

$$
E_{\text {free }}=\pi \kappa \frac{a}{\lambda}\left(\frac{k^{3}}{1-k^{2}}\right) \frac{K_{0}(k a / \lambda)}{K_{1}(k a / \lambda)},
$$

where we introduced the abbreviation $k=\sqrt{z(2-z)}$.

The small gradient expansion and the exact membrane profiles are shown in Fig. 囵 for different values of the detachment angle. As expected, the quality of the approximation declines as the degree of penetration increases (note that the parameterization itself fails much later, namely for $z>1$ ). To further illustrate the discrepancy between the exact and the approximate solution, we plot in Fig. 5 the energy of the free part of the membrane. 


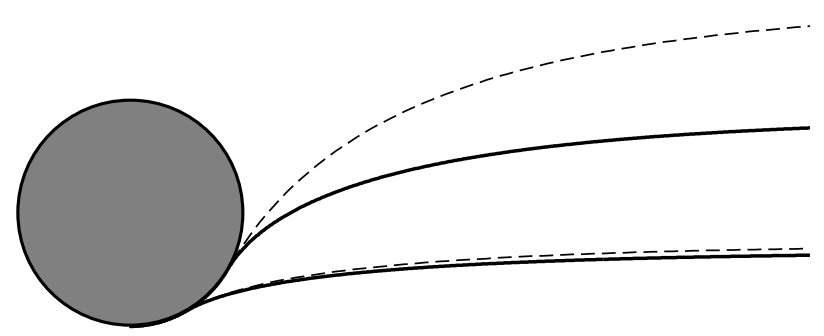

FIG. 4: Exact membrane profiles (solid curves) and small gradient approximation (dashed curves) for two prescribed detachment angles $\alpha=30^{\circ}$ and $\alpha=60^{\circ}$ for the tension $\sigma a^{2} / \kappa=0.1$.

The inset shows the energy difference between the small gradient expansion and the nonlinear result. We see that the approximation is fairly reasonable for degrees of penetration up to $z \approx 0.1$. Beyond this value, one has to solve the full shape equation in order to give an accurate description of the deformation energy.

Nevertheless, the small-gradient expansion improves our understanding of the system, since it allows us to work out the asymptotic phase boundaries. We consider first the transition between the free and the partially wrapped state. For small degrees of penetration the total energy of the system can be expanded up to quadratic order in $z$, giving

$$
\begin{aligned}
E=\pi a^{2}\{- & 2 z\left(w-w_{\mathrm{c}}\right)+ \\
& \left.\sigma z^{2}\left[1-4 \gamma-2 \ln \frac{\sigma z}{w_{\mathrm{c}}}\right]+\mathscr{O}\left(z^{2}\right)\right\},
\end{aligned}
$$

where $\gamma=0.5772 \ldots$ is the Euler-Mascheroni constant [17. For sufficiently small $z$ the quadratic term is negligible and wrapping sets in as soon as the prefactor of the linear term becomes negative. This confirms the numerical finding that the initial wrapping transition is continuous and always takes place at $w=w_{\mathrm{c}}$, no matter what the value of the tension is.

Even though the final state of the second transition toward full envelopment cannot be described within a small gradient expansion, its energy is exactly known, since the free membrane does not contribute to it. The location of this second transition can therefore also be predicted by equating the energy of the partially wrapped state, eq. (7), with the energy of the fully enveloped state, $E=4 \pi a^{2}(\sigma-w)+8 \pi \kappa$. Since this yields a transcendental equation, the relation between $\sigma$ and $w$ on the phase boundary cannot be expressed in terms of simple functions. However, the asymptotic behavior at zero tension, which remains quite accurate over the whole regime of low tension, is surprisingly simple: We find that, up to a complicated but small logarithmic correction, the transition is given by $\sigma=w-w_{\mathrm{c}}$ (see Fig. 2).

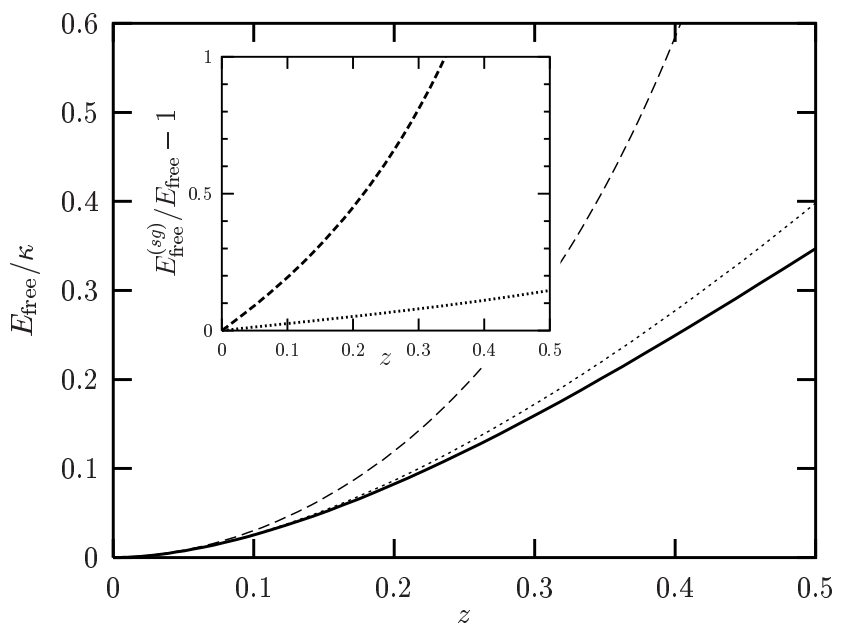

FIG. 5: Energy $E_{\text {free }}$ of the free part of the membrane for the tension $\sigma a^{2} / \kappa=0.1$ as a function of the degree of wrapping, $z$. Solid, dashed, and dotted curves correspond to the full nonlinear solution, the small gradient approximation, and its quadratic expansion, respectively. The inset shows the relative error of the two small gradient expressions.

Unfortunately, the concomitant energy barrier cannot be obtained in small gradient expansion. Even if the transition toward envelopment takes place at small $z$, where the approximation still works, the barrier occurs at large penetrations, $z>1$ (see $e . g$. inset of Fig. 3). This is even beyond the range of applicability of the Monge parameterization $h=h(x, y)$.

\section{DISCUSSION}

The combination of both numerical an analytical approaches presented in this work has provided a complete description of the equilibrium wrapping behavior. The structure of the wrapping complex, the transition lines in the wrapping diagram, and the barriers of the discontinuous envelopment boundary can be predicted, and the low tension regime can even be treated analytically. One particular consequence of the wrapping scenario is that colloid engulfment is extremely sensitive to the particle size. As long as the tension is low enough $(\sigma<w / 2$ will do), a large sphere is wrapped much more easily than a small one. This could be checked experimentally for instance by spreading a bilayer across a hole separating two compartments and adding a polydisperse colloidal solution to one side. If there exists some generic attraction between the colloids and the bilayer, a population of bilayer-coated colloids should emerge in the other compartment with a different polydispersity distribution: The frequency of large colloids is significantly enhanced, and too small ones do not occur at all.

An important issue that we have not addressed here is the effect of thermally excited membrane fluctuations. At very low tension, the undulations might prevent the 
beads from adhering to the membrane. An initial study of finite-size effects on the Helfrich repulsion shows that the (entropic) barrier to overcome in order to reach the surface increases with particle size [18]. This would provide an interesting counterbalance to the tendency to engulf preferentially large spheres.

The level of description in this work is that of generic physical mechanisms, but several examples mentioned in the introduction are taken from biology, where a host of different effects often occur at the same time. Notwithstanding this difficulty, we believe it to be worthwhile to analyze these examples - in particular, viral budding in terms of the mechanisms discussed here. For instance, the adhesion strength of viral capsids to membranes can be modified by changing the biochemistry or simply the chemical potential of the binding proteins which in most cases are responsible for the attachment. Moreover, the membrane tension of cells increases or decreases during times of heightened endo- or exocytosis activity, respec- tively, and is generally under active control of the cell [11]. Thus, processes like viral budding and its later counterpart of unwrapping (after infection of a different host cell and fusion with its membrane) invariably involve a movement in the structural phase diagram of Fig. 2 2 along both axes. The concomitant "phase changes" predicted that way thus offer an understanding of the observed (un)wrapping processes in terms of the physical forces driving them.

\section{Acknowledgments}

We would like to thank Bill Gelbart, Shelly Tzlil, and Avinoam Ben-Shaul for many stimulating discussions on the subject. MD also gratefully acknowledges financial support of the German Science Foundation under grant De775/1-1.
[1] Lodish H., Berk A., Zipursky S. L., Matsudaira P., Baltimore D., and Darnell J., Molecular Cell Biology, Freeman \& Company, New York (2000).

[2] Garoff H., Hewson R., and Opstelten D.-J. E., Microbiol. Mol. Biol. Rev. 62, 1171 (1998).

[3] Boussif O., Lezoualc'h F., Zanta M. A., Mergny M. D., Scherman D., Demeneix B., and Behr J.-P., Proc. Nat. Acad. Sci. 92, 7297 (1995).

[4] Caspi A., Yeger O., Grosheva I., Bershadsky A. D., and Elbaum M., Biophys. J. 81, 1990 (2001).

[5] Henderson R. M. and Oberleithner H., Am. J. Physiol. Renal Physiol. 278, F689 (2000).

[6] Dietrich C., Angelova M. and Pouligny B., J. Phys. II (France) 7, 1651 (1997).

[7] Koltover I., Rädler J. O., and Safinya C. R., Phys. Rev. Lett. 82, 1991 (1999).

[8] Deserno M. and Gelbart W. M., J. Phys. Chem. B 106,
5543 (2002).

[9] Boulbitch A., Europhys. Lett. 59, 910 (2002).

[10] Helfrich W., Z. Naturforsch. 28c, 693 (1973).

[11] Morris C. E. and Homann U., J. Membrane Biol. 179, 79 (2001).

[12] Baker T. S., Olson N. H., and Fuller S. D., Microbiol. Mol. Biol. Rev. 63, 862 (1999).

[13] Seifert U., Adv. Phys. 46, 13 (1997).

[14] Jülicher F. and Seifert U., Phys. Rev. E, 49, 4728 (1994).

[15] Deserno M., manuscript in preparation.

[16] Fourcade B., Miao L., Rao M., Wortis M., and Zia R. K. P., Phys. Rev. E 49, 5276 (1994).

[17] Abramowitz M. and Stegun I. A. (eds.), Handbook of mathematical functions, Dover, New York (1970).

[18] Bickel T., submitted. 